\title{
FIR and Submillimeter Optical Properties of Astrophysically relevant Minerals
}

\author{
Karine Demyk*, Claude Meny \\ Université de Toulouse; UPS-OMP; IRAP; Toulouse, France \\ CNRS; IRAP; 9 Av. colonel Roche, BP 44346, F-31028 Toulouse cedex 4, France \\ E-mail: karine.demykeirap. omp.eu
}

Christophe Depecker, Hugues Leroux

UMET, UMR 8207, Université Lille 1, CNRS, F-59655 Villeneuve d'Ascq, France

Céline Nayral, Fabien Delpech, Willfried-Solo Ojo

Université de Toulouse, INSA, CNRS, LPCNO, F-31077 Toulouse, France

\section{Jean-Blaise Brubach, Pascale Roy}

Ligne AILES - Synchrotron SOLEIL, L'Orme des Merisiers, F-91192 Gif-sur-Yvette, France

\begin{abstract}
The Herschel and Planck missions have opened up the spectral domain in the far infrared and submillimeter range. This spectral domain reveals the emission of cold ( $\mathrm{T} \leq 50 \mathrm{~K})$ dust grains of nanometer size $(10-100 \mathrm{~nm})$, emission which is used to study star formation, to estimate interstellar clouds masses and more generally to study the evolution of the interstellar medium in our Galaxy and in external galaxies. To get reliable interpretation of the observations it is therefore crucial to properly understand the physic of dust emission and to know as well as possible the FIR and submm optical properties of interstellar dust. In this article we will review the theoretical and experimental works aiming at studying the physics of dust analogues in the FIR/submm domain. The mass absorption coefficient of amorphous silicate nano grains decreases with the temperature and shows a complex shape with the wavelength depending on the micro structure of the materials. For wavelengths shorter than $\sim 500 \mu \mathrm{m}$ the spectral index is in the range 1.6-2.3 whereas at longer wavelengths it changes and its value may be outside this range. Therefore, a single spectral index is not always adequate to correctly fit the experimental measurements in the $100-1000 \mu \mathrm{m}$ domain. These results must be taken into account in astronomical modeling either by using optical constants measured in the FIR/submm domain and at low temperature or by using physical models able to reproduce the experimental data.
\end{abstract}

The Life Cycle of Dust in the Universe: Observations, Theory, and Laboratory Experiments - LCDU 2013, 18-22 November 2013

Taipei, Taiwan

\footnotetext{
* Speaker.
} 


\section{Dust emission in the FIR/submm domain: observations and dust modeling}

In cold environments such as the Interstellar Medium (ISM), dust grains in thermal equilibrium with the radiation field emit in the far infrared and submillimeter domain (FIR/submm). In this spectral domain, the optical depth being generally thin and assuming local thermodynamic equilibrium, the dust emission is usually modeled as the emission of a modified blackbody. The specific intensity $\mathrm{I}_{v}$ is then, expressed as:

$$
\mathrm{I}_{v}(\lambda)=\mathrm{B}(\lambda, \mathrm{T}) \mathrm{N}_{\mathrm{H}} \mathrm{Q}_{\mathrm{abs}}(\lambda)
$$

where $\mathrm{B}(\lambda, \mathrm{T})$ is the Planck function at the dust temperature $\mathrm{T}, \mathrm{Q}_{\mathrm{abs}}(\lambda)$, the dust absorption efficiency and $\mathrm{N}_{\mathrm{H}}$ the hydrogen column density. In the FIR/submm range, $\mathrm{Q}_{\mathrm{abs}}(\lambda)$ is usually extrapolated by a simple power law of the wavelength and it expression is :

$$
\mathrm{Q}_{\mathrm{abs}}(\lambda)=\varepsilon_{0}\left(\lambda / \lambda_{0}\right)^{-\beta}
$$

where $\varepsilon_{0}$ is the emissivity at the reference wavelength $\lambda_{0}$ and $\beta$ the spectral index. Simple semiclassical physical models describing the interaction of dust with light, such as the Lorentz model, predict that the spectral index, $\beta$, is equal to 2 at long wavelength, a value commonly adopted in astrophysical studies [1]. Knowing the value of the spectral index or adopting $\beta=2$, it is thus straightforward to derive the dust mass and temperature and hence the cloud mass from the observations. However it has long been known that this simple model does not explain many observations. The spectral index derived from the observations, the emissivity spectral index $\beta_{e m}$, is generally different than 2 in astrophysical models [2-4]. In addition, the FIR excess observed at wavelength larger than $500 \mu \mathrm{m}$ and the so-called $\beta_{e m}$-T anti-correlation have shown that the observed spectral emissivity index $\beta_{e m}$ may change with frequency and with the grain temperature $[5,6]$.

First observed by Reach et al. [5], the FIR excess is now widely observed by Herschel and Planck in our Galaxy ([6] and reference therein) and in external galaxies such as the LMC ([7] and reference therein). Attempts to model the FIR excess fall in two categories, one that uses the modified blackbody emission model but adopting new dust components, such as amorphous carbon instead of graphite [7] or magnetic nanoparticles [8], the other that uses alternative dust emission models such as the TLS model $[1,9,6]$.

Dupac et al. [2] followed by Désert et al. [3] observed in many cold regions of the ISM that the spectral index $\beta_{e m}$ is anti-correlated with the dust temperature. Many additional observational studies have recently found similar anti-correlation and values for $\beta_{e m}$ than can reach more than 3 at very low dust temperatures and around 1 for warmer temperatures $[4,10]$. Although $\beta$ values greater than 2 contradict the Lorentz model, it is compatible with the TLS model [1]. However, when derived by modeling the observations with a modified blackbody, $\beta$ and $\mathrm{T}$ are partially degenerate and present an anti-correlation. It was demonstrated that the determination of $\beta$ is influenced by the method used to fit the SED [11, 12]. The chi-square method tends to accentuate the anticorrelation whereas other methods such as the SIMEX methods attenuates it. In addition, it was shown that an anti-correlation between $\mathrm{T}$ and $\beta$ might be induced for regions containing embedded hot sources [13]. The interpretation of the $\beta$-T anti-correlation is thus complicated and should 
consider the fitting method used and radiative transfer modeling.

\section{Optical characterization of interstellar dust analogues in the FIR/submm domain}

The grains responsible for the observed FIR/submm emission are large enough to be in thermal equilibrium, and not stochastically heated by the radiation field, i.e. they have an average size of $\sim 15 \mathrm{~nm}$ up to several hundreds of $\mathrm{nm}$. They are composed of silicates and carbonaceous matter in separate and/or composite grains made of a silicate core covered with carbonaceous matter [14]. Their composition is inferred from the comparison of spectroscopic observations in the midinfrared domain, where the chemical bonds constituting the grain vibrates, with spectra of dust analogues synthesized in the laboratory. Above $\sim 100 \mu \mathrm{m}$, there are no more vibrational bands and the interaction of matter with light is due to single or multi-phonon processes that produces a continuum of absorption [15, 16] or to the low lying excitation spectrum of two level systems (TLS) in glasses [17, 1].

The modeling of the dust emission at FIR/submm wavelengths relies, among other things, on the a priori knowledge of the dust optical properties in this domain. Numerous experimental studies have been undertaken to characterize the optical properties of silicate dust analogues in the mid infrared spectral range (see for example [18] and associated papers). In some cases, experimental spectra were recorded up to 100 or $300 \mu \mathrm{m}$, and sometimes at low temperatures [19]. However, only a few laboratory studies have been dedicated to the FIR/submm spectral range and to low temperatures (see Table 1 and reference therein). However, despite the existence of experimental data at these long wavelengths, the dust optical properties used in astronomical modeling are extrapolated from those measured at shorter wavelengths [20,21]. In the framework of the Lorentz theory, this extrapolation is expressed as a power law of the wavelengths, with a spectral index $\beta=$ 2 or in the range 1-2 at all wavelength and for all temperatures [1].

Agladze et al. [17] measured the absorption spectra of Mg-rich amorphous and crystalline silicates at long wavelengths $(700 \mu \mathrm{m}-2.9 \mathrm{~mm})$ and very low temperatures $(1.2-30 \mathrm{~K})$. They found the mm mass absorption coefficients (MAC) of the amorphous materials differed from the values of the astrosilicates from [22] by factors of 0.95 to 11 depending on the synthesis method and consequently on the microscopic structure of the materials. They also observed that the spectral index describing the mass absorption coefficient in the $700-2900 \mu \mathrm{m}$ range varied in the range 1.52.5 depending on the material and that it was anti correlated with the temperature in the range 10-30 $\mathrm{K}$, this variation being more pronounced for amorphous material than for crystalline samples. They attributed the temperature dependence of the FIR mass absorption coefficient to resonant absorption by glassy two-level systems, the so-called TLS model [23-25]. Additional measurements were performed by Mennella et al. [15] at shorter wavelength $(20-2000 \mu \mathrm{m})$ and higher temperature $(24-300 \mathrm{~K})$ on forsterite $\left(\mathrm{Mg}_{2} \mathrm{SiO}_{4}\right)$ and amorphous and crystalline fayalite $\left(\mathrm{Fe}_{2} \mathrm{SiO}_{4}\right)$. They also observed that the spectral index describing the MAC is anti-correlated with the temperature and the MAC decrease with decreasing temperature. They proposed that these effects, which are more important for amorphous material, should be principally due to two-phonons difference processes. In the same study, they also measured the MAC of amorphous carbon samples finding similar 


\begin{tabular}{|c|c|c|c|c|c|}
\hline Material & $\begin{array}{c}\text { Spectral } \\
\text { domain }(\mu \mathrm{m})\end{array}$ & $\begin{array}{c}\text { Temperature } \\
\text { range }(\mathrm{K})\end{array}$ & $\begin{array}{c}\beta \text { value } \\
10 \mathrm{~K}-300 \mathrm{~K}\end{array}$ & $\begin{array}{c}\kappa(1 \mathrm{~mm}) \\
10 \mathrm{~K}-300 \mathrm{~K}\end{array}$ & Reference \\
\hline am. $\mathrm{Na}_{2} \mathrm{O}-\mathrm{CaO}-\mathrm{SiO}_{2}$ & $500-5000$ & $300-0.04$ & $3-1.6$ & & Bösch [24] \\
\hline am. $2 \mathrm{MgO}-\mathrm{SiO}_{2}$ & \multirow[b]{2}{*}{$700-2900$} & \multirow[b]{2}{*}{$30-1.2$} & \multirow[b]{2}{*}{$2.5-2$} & $0.26 / 1.56$ & \multirow[b]{2}{*}{ Agladze et al. [17] } \\
\hline $\begin{array}{c}\text { am. } \mathrm{MgO}-\mathrm{SiO}_{2} \\
\text { crys. } \mathrm{Mg}_{2} \mathrm{SiO}_{4} \\
\text { crys. } \mathrm{MgSiO}_{3}\end{array}$ & & & & $\begin{array}{l}0.28 \\
0.23 \\
0.08\end{array}$ & \\
\hline crys. $\mathrm{Mg}_{1.8} \mathrm{Fe}_{0.2} \mathrm{Si}_{2} \mathrm{O}_{4}$ & \multirow{5}{*}{$20-200$} & \multirow{5}{*}{$300-24$} & $2.2-2$ & $0.16-0.43$ & \multirow{5}{*}{ Mennella et al. [15] } \\
\hline crys. $\mathrm{Mg}_{0.1} \mathrm{Fe}_{1.9} \mathrm{Si}_{2} \mathrm{O}_{4}$ & & & $2.3-2$ & $0.21-0.41$ & \\
\hline am. $\mathrm{Mg}_{0.2} \mathrm{Fe}_{1.8} \mathrm{Si}_{2} \mathrm{O}_{4}$ & & & $2.3-2$ & $0.86-5.0$ & \\
\hline am. carbon BE & & & $1.2-0.9$ & $21-72$ & \\
\hline am. carbon ACAR & & & $1.1-0.8$ & $26-69$ & \\
\hline am. $\mathrm{SiO}_{2} 1.5 \mu \mathrm{m}$ & \multirow{4}{*}{$100-2000$} & \multirow{4}{*}{$300-10$} & $2.8-1.1 / 1.3-0.9^{(a)}$ & $0.33-3.75$ & \multirow{4}{*}{ Boudet et al. [16] } \\
\hline am. $\mathrm{SiO}_{2}$ fused & & & $2.4-1.1 / 1.3-1.1^{(a)}$ & $0.45-3.75$ & \\
\hline am. $\mathrm{MgSiO}_{3}$ glass & & & $2.1-1.6 / 1.6-1.2^{(a)}$ & $0.22-0.75$ & \\
\hline am. $\mathrm{MgSiO}_{3}$ solgel & & & $2.7-1.4 / 1.9-1.7^{(a)}$ & $0.12-0.98$ & \\
\hline am. $\mathrm{Mg}_{2.3} \mathrm{SiO}_{4}$ & \multirow{5}{*}{$100-1500$} & \multirow{5}{*}{$300-10$} & $3.6-2.2 / 2.1-1.6^{(b)}$ & $1.36-5.22$ & \multirow{5}{*}{ Coupeaud et al. [26] } \\
\hline am. $\mathrm{Mg}_{2.8} \mathrm{SiO}_{4}$ & & & $3.2-2.5 / 2.1-1.8^{(c)}$ & $2.14-4.95$ & \\
\hline am. $\mathrm{Mg}_{2.05} \mathrm{SiO}_{4}$ & & & $4.5-2.5 / 1.9-1.9^{(d)}$ & $0.78-2.04$ & \\
\hline am. $\mathrm{Mg}_{0.95} \mathrm{SiO}_{3}$ & & & $2.5-1.7 / 1.7-1.3^{(e)}$ & $1.36-9.19$ & \\
\hline am. $\mathrm{Ca}_{0.98} \mathrm{Mg}_{0.9} \mathrm{Si}_{2} \mathrm{O}_{6}$ & & & $2-1.8 / 1.4-1.3^{(f)}$ & $6.98-10.74$ & \\
\hline am. $\mathrm{Mg}_{0.8} \mathrm{Fe}_{0.2} \mathrm{SiO}_{3}-\mathrm{Fe}^{3+}$ & \multirow{4}{*}{$100-1000$} & \multirow{4}{*}{$300-10$} & $2.7-1.5 / 2.0-1.4^{(g)}$ & $0.45-1.83$ & \multirow{4}{*}{ Demyk et al. in prep. } \\
\hline am. $\mathrm{Mg}_{0.8} \mathrm{Fe}_{0.2} \mathrm{SiO}_{3}-\mathrm{Fe}^{2+}$ & & & $1.7-1.5 / 1.5-1.2^{(g)}$ & $2.29-3.92$ & \\
\hline am. $\mathrm{Mg}_{0.9} \mathrm{Fe}_{0.1} \mathrm{SiO}_{3}-\mathrm{Fe}^{3+}$ & & & $2.6-2.0 / 2.1-1.2^{(h)}$ & $0.09-0.98$ & \\
\hline am. $\mathrm{Mg}_{0.9} \mathrm{Fe}_{0.1} \mathrm{SiO}_{3}-\mathrm{Fe}^{2+}$ & & & $2.2-1.4 / 1.8-1.2^{(i)}$ & $1.31-3.23$ & \\
\hline
\end{tabular}

Table 1: Experimental studies on astrophysically relevant dust analogues in the FIR/submm/mm domain at low temperature. The value of spectral index is derived on the whole spectral domain except for (a): 500 - $1000 \mu \mathrm{m}$ and $200-100 \mu \mathrm{m}$ respectively, (b): $700-1200 \mu \mathrm{m}$ and $130-700 \mu \mathrm{m}$, (c): $785-1000 \mu \mathrm{m}$ and $170-785 \mu \mathrm{m},(\mathrm{d}): 600-1200 \mu \mathrm{m}$ and $150-600 \mu \mathrm{m},(\mathrm{e}): 500-800 \mu \mathrm{m}$ and $150-500 \mu \mathrm{m}$, (f): $450-650 \mu \mathrm{m}$ and $150-450 \mu \mathrm{m},(\mathrm{g}): 200-1000 \mu \mathrm{m}$ and $100-200 \mu \mathrm{m}$, (h): $200-700 \mu \mathrm{m}$ and $100-200 \mu \mathrm{m}$, (i): $200-830 \mu \mathrm{m}$ and $100-200 \mu \mathrm{m}$. Note that Coupeaud et al. have also studied crystallized samples obtained by annealing of the amorphous samples presented in this table, see [26] for the values of $\beta$ and $\kappa(1 \mathrm{~mm})$. 

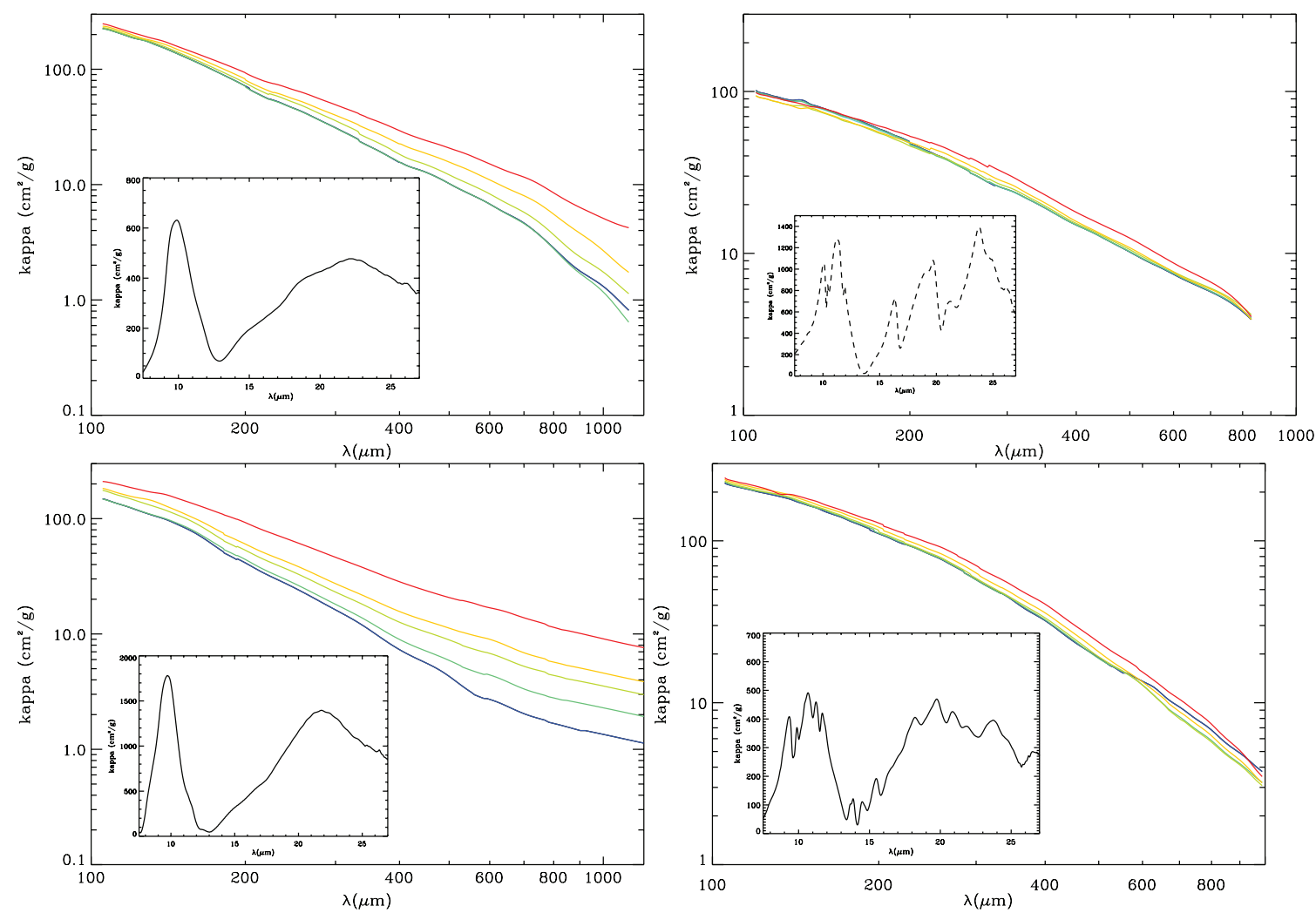

Figure 1: Mass absorption coefficients of Mg-rich silicates from Coupeaud et al. [26] as a function of the temperature (red: $300 \mathrm{~K}$, orange: $200 \mathrm{~K}$, green: $100 \mathrm{~K}$, light blue : $30 \mathrm{~K}$, dark blue: $10 \mathrm{~K}$ ). Upper panels: $\mathrm{Mg}_{2.3} \mathrm{SiO}_{4}$ solgel samples, amorphous (left panel) and crystalized by annealing at $1100^{\circ} \mathrm{C}$ for $24 \mathrm{~h}$ (right panel). Lower panels: $\mathrm{Mg}_{0.95} \mathrm{SiO}_{3}$ solgel samples, amorphous (left panel) and crystallized by annealing at $1100^{\circ} \mathrm{C}$ for $24 \mathrm{~h}$ (right panel). The insets shows the MIR spectra of the samples at room temperature.

trends with temperature. However the values derived for the spectral indexes are in the range 0.8 - 1.2 (depending on the temperature and on the material), significantly lower than for their silicate samples $(1.4-2.4)$. They thus proposed that silicate grains coated with amorphous carbon should be considered in astronomical models.

With the aim of extending the samples of studied materials new experimental studies were performed by Boudet al. [16] on amorphous silicates having a composition of $\mathrm{MgSiO}_{3}$ and on silica $\left(\mathrm{SiO}_{2}\right)$ sub-micron grains in the 7- $1500 \mu \mathrm{m}$ range and at low temperature (10-300 K). This experimental effort was continued by Coupeaud et al. [26] on olivine and pyroxene like amorphous and crystallized samples using synchrotron radiation to extend the measurements to the submillimeter range [27]. In agreement with previous studies, Boudet et al. and Coupeaud et al. observed for the amorphous samples a clear decrease of the MAC with decreasing temperature and, at a given wavelength, an increase of the spectral index with decreasing temperature. However, when the amorphous samples were annealed up to crystallization the variations of the MAC and of $\beta$ with the temperature were not observed (Figure 1). However, in contrast with previous studies they observed that the absorption coefficients of the amorphous samples are not only temperaturedependent but also frequency-dependent (Figure 1). Therefore, several spectral indexes are defined 

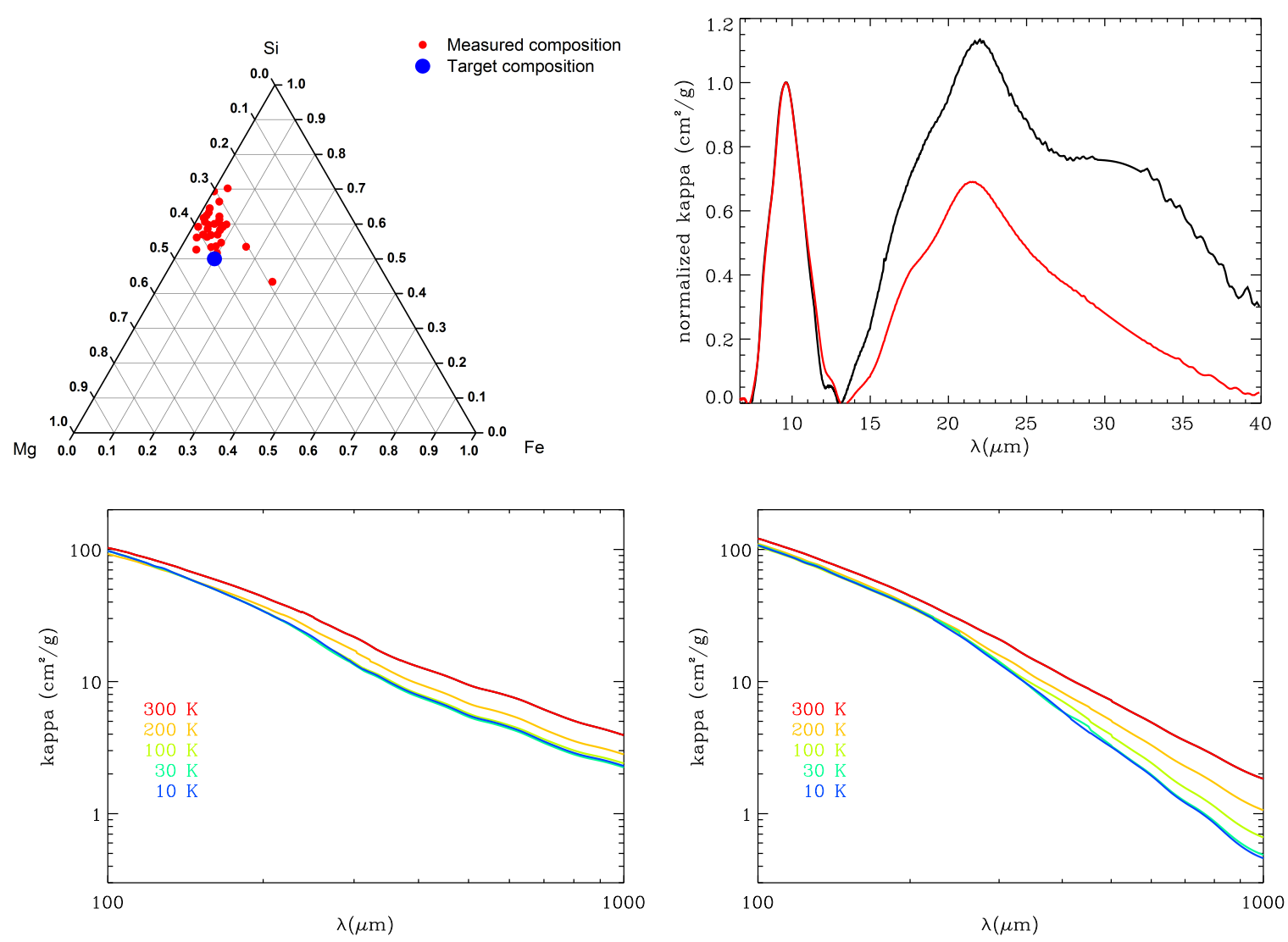

Figure 2: Mass absorption coefficient of amorphous samples with a mean composition $\mathrm{Mg}_{0.8} \mathrm{Fe}_{0.2} \mathrm{SiO}_{3}$. Left upper panel: measured composition. Right upper panel: normalized MAC measured at room temperature in the 8-40 $\mu \mathrm{m}$ range of sample containing $\mathrm{Fe}^{3+}$ (black curve) and of sample containing $\mathrm{Fe}^{2+}$ (red curve). Lower panels: MAC measured in the $100 \mu \mathrm{m}-1 \mathrm{~mm}$ range at different temperatures (red: $300 \mathrm{~K}$, orange: $200 \mathrm{~K}$, green: $100 \mathrm{~K}$, light blue : $30 \mathrm{~K}$, dark blue: $10 \mathrm{~K}$ ) for the $\mathrm{Fe}^{2+}$-rich sample (left) and the $\mathrm{Fe}^{3+}$-rich sample (right).

on spectral domains that vary according to the samples but also to the temperature (see Table 1 and Figures 6 and 7 in [16] and Table 3 in [26]).

Except for the amorphous fayalite sample (Mennela et al. 1998), no iron-rich material has been studied. Yet, iron, being highly depleted from the gas phase, is an essential component of cosmic dust, even though the way it is incorporated into grains is unknown. Iron could be in the form of iron oxide or metallic iron inclusions within the silicate grains or as a separate grain population. It could also be part of the silicate amorphous network in the form of $\mathrm{Mg}$-Fe-rich silicates. We present here new results on amorphous $\mathrm{Mg}$ and $\mathrm{Fe}$ silicates of pyroxene composition $\mathrm{Mg}_{1-x} \mathrm{Fe}_{x} \mathrm{O}_{3}$, with $\mathrm{x}$ $=0.1$ and $\mathrm{x}=0.2$. The oxidation state of the Fe ions resulting from the solgel synthesis is $\mathrm{Fe}^{3+}$. However, Fe may also be present within the silicate in the form of $\mathrm{Fe}^{2+}$. To change the oxidation state of iron we have annealed the $\mathrm{Fe}^{3+}$ samples at $500^{\circ} \mathrm{C}$ for two hours in a reducing atmosphere. Although we have not quantified the amount of $\mathrm{Fe}^{2+}$ in the "reduced" samples the MIR spectra of the normal and reduced samples shown in Figure 2 clearly show that the spectral band at $\sim 33$ 
$\mu \mathrm{m}$ attributed to the vibration of iron has disappeared in the reduced sample. The MAC of the two samples (Figure 2) presents the same dependence on the temperature as those of iron free silicates from previous studies and to the one of amorphous $\mathrm{Fe}_{2} \mathrm{Si}_{2} \mathrm{O}_{4}$ from [15]: the MAC decreases with the temperature and the spectral index is higher at lower temperature. The spectral shape of the MAC is complex and different for the $\mathrm{Fe}^{3+}$ and $\mathrm{Fe}^{2+}$ sample. The MAC is flatter and the variations with temperature are smaller for the reduced, $\mathrm{Fe}^{2+}$, sample whereas it is steeper and variations are larger for the $\mathrm{Fe}^{3+}$ sample. We have observed the same trend for the sample containing $10 \%$ of iron $\left(\mathrm{Mg}_{0.9} \mathrm{Fe}_{0.1} \mathrm{SiO}_{3}\right)$. By changing the oxidation state of iron, the silicate network arrangement at atomic scale is transformed, which, in turn, may modify the absorption in the FIR domain. To verify the hypothesis that the different shape of the MAC is related to the oxidation state of iron we will study two additional samples containing 30 and $40 \%$ of iron (Demyk et al. in prep).

\section{Astrophysical applications}

From Table 1 it appears that in the Herschel spectral range $(100-500 \mu \mathrm{m}), \beta$ is in the range 1.6 - 2.3 for all the silicate samples and that the variation of $\beta$ with temperature is smaller than at longer wavelength. The scatter in the $\beta$ value may arise from differences in the composition and structure of the materials that have been synthesized with various techniques (solgel, laser pulverization, quenching method). The spectral shape of the MAC changes in the 400-600 $\mu \mathrm{m}$ range, depending on the sample and on the temperature $[16,26]$. At wavelengths longer than $\sim 500 \mu \mathrm{m}$, $\beta$ may have more extreme values, larger than 2.5 and smaller than 1.5 . Consequently the decrease of the MAC from $300 \mathrm{~K}$ to $10 \mathrm{~K}$ is more important at longer wavelength than at shorter wavelengths. These experimental results have some implications for the interpretation of astronomical observations. Dust column density and cloud mass being proportional to the MAC used in astrophysical models, the use of MAC measured or extrapolated from data taken at room temperature will induce larger errors on mass determination at long wavelengths than at shorter (Herschel) wavelengths. In addition, the use of a single spectral index on the whole spectral domain, whereas experimental results show it may change above $\sim 500 \mu \mathrm{m}$ even at low temperature, may introduce large errors on the mass determination at millimeter wavelengths.

Another difficulty arises from the fact that interstellar dust is composed of a variety of compositions and amorphous structures that all contribute to the FIR/submm emission in proportion to the abundance of the different components. The emissivity spectral index derived from the modeling of astronomical observations is therefore an average spectral index that should not be compared with the experimental results for a single dust analogue. Likewise, astronomical models based on the optical properties of interstellar dust analogues should consider several dust components. Dust models are generally composed of at least two grains components: a population of silicate grains with a single composition and a population of carbon grains composed of graphite or of amorphous carbon. Although there are only a few measurements of carbonaceous matter in the FIR/submm at low temperature, it appears that amorphous carbon samples are much more absorbing than the silicates samples and they have a very different spectral shape, much flatter than that of silicate materials for all temperatures (Table 1). Large carbon grains may thus contribute significantly to the observed FIR/submm emission even though they do not dominate in mass. 


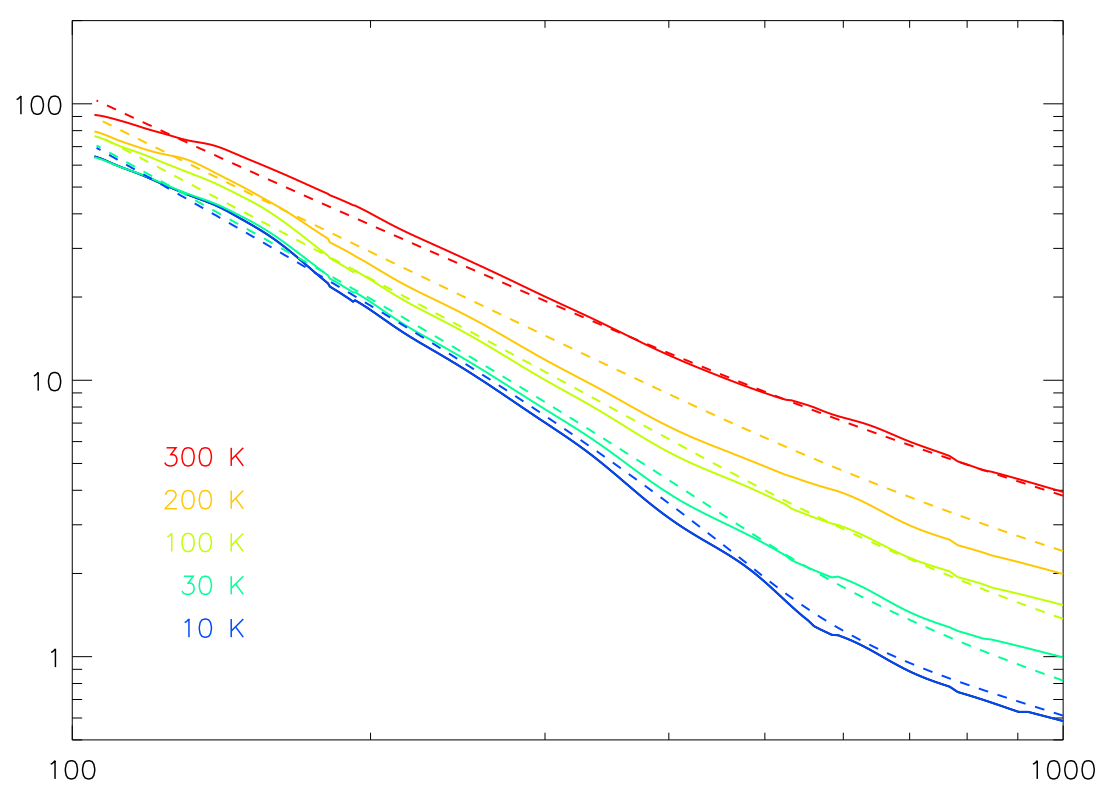

Figure 3: Fit of the MAC of amorphous MgSiO3 from [26] (dashed lines) with the TLS model from [1] (solid lines at various temperature).

The spectral shape of the MAC and its variations with temperature reflect the coupling of light to the amorphous structure of the material. Two mechanisms were proposed to explain this behavior that applies to all amorphous material with a large degree of universality. The first one describes local defects and disorder at atomic scale within the grains by a single charge correlation length (DCD model, [23]) and the second one by an ordered distribution of Two-levels systems (TLS model, [24]). They have been coupled into a single model adapted to astronomical studies, the TLS model [1]. The TLS model reproduces the experimental data (Figure 3) but also the astronomical observations. Three sets of parameters have been derived from the observations of the diffuse, dense and standard ISM [9]. They characterize the mean properties of the dust in these environments, averaged on the different dust components. They can be used as input to the TLS model to predict and model the dust emission of these environments at different temperatures in the FIR domain.

\section{Conclusion and perspectives}

Experimental data on analogues of interstellar silicates are now numerous and cover a large range of composition and structure. All amorphous materials show a universal behavior in the FIR/submm domain with the temperature: their MAC decrease with the temperature and at aiven wavelength, the spectral index increases when the grain temperature decreases. The few experimentally studied amorphous carbon materials behave the same and since their MAC are much larger than the MAC of silicate analogues investigated so far, it seems important to extend experimental studies to additional carbonaceous materials. Below $500 \mu \mathrm{m}$, the spectral index $\beta$ of the 
silicates samples is in the range 1.6 - 2.3 for all temperatures whereas at longer wavelength it can be as low as 1 or as high as 3 and more, depending on the material. These variations are due to the amorphous structure of the material and may be modeled using the TLS model which also succeed in reproducing Herschel observations. It is important to take into account these experimental results in astronomical modeling in order to minimize the error on the column density and mass determination but also for a proper understanding of the evolution of the dust properties and of the host environment.

Acknowledgement: This work has been supported by the French National Agency (ANR-11-BS56-0029, "CIMMES: Cold Insterstellar Matter: modeling- and experimental-based studies"), by the European programme FEDER and by the programme "Molécules et grains : du laboratoire l'Univers" of the MidiPyrénées Observatory. We acknowledge SOLEIL for provision of synchrotron radiation facilities.

\section{References}

[1] Meny C., Gromov V., Boudet N., et al., 2007, A\&A 468, 171

[2] Dupac X., Bernard J., Boudet N., et al. 2003, A\&A, 404, L11

[3] Désert F., Macías-Pérez J.-F., Mayet F., et al. 2008, A\&A, 481, 411

[4] Rodón J. A., Zavagno A., Baluteau J.-P., et al., A\&A, 518, L80

[5] Reach W.T., Dwek E., Fixsen D. J., et al. 1995, ApJ, 451, 188

[6] Paradis D., Paladini R., Noriega-Crespo A., et al., 2012, A\&A, 537, A113

[7] Galametz M., Hony S., Galliano F., et al., 2013, MNRAS, 431, 1596

[8] Draine B. T., Hensley B., 2012, ApJ, 757, 103

[9] Paradis D., Bernard J.-P., Meny C., Gromov V., 2011, A\&A, 534, A118

[10] Planck Collaboration, 2011, A\&A, 536, A22

[11] Juvela M., Montillaud J., Ysard N., Lunttila T., 2013, A\&A 556, A63

[12] Juvela M. \& Ysard N., 2012, A\&A 539, A71

[13] Ysard N., Juvela M., Demyk, K., et al., A\&A, 542, A21

[14] Compiègne M., Verstraete L., Jones A., et al., A\&A, 525, A103

[15] Mennella V., Brucato J. R., Colangeli L., et al. 1998, ApJ, 496, 1058

[16] Boudet N., Mutschke H., Nayral C., et al., 2005, ApJ, 633, 272

[17] Agladze N. I., Sievers, A. J. Jones, et al., 1996, ApJ, 462, 1026

[18] Jaeger C., Mutschke H., Begemann B., Dorschner J., Henning Th., 1994, A\&A, 292, 641

[19] Koike C., Mutschke H., Suto, H., et al., 2006, A\&A, 449, 583

[20] Draine, B.T., 2003, ApJ, 598, 1026

[21] Zubko V.G., Mennella, V., Colangeli L. \& Bussoletti E., 1996, MNRAS, 282, 1321

[22] Draine B. T., Lee H. M., 1984, ApJ, 285, 89 
[23] Schlömann E., 1964, Phys. Rev., 133, A413

[24] Bösch M.A., 1978, Phys. Rev. Lett., 40, 879

[25] Phillips W.A., 1987, Rep. Prog. Phys., 50, 1657

[26] Coupeaud A., Demyk K., Meny C., et al. 2011, A\&A, 535, A124

[27] Brubach J. B., Manceron L., Rouzière M., et al., AIP Conference Proceedings, 2010, 1214: 81-84 